\title{
HEPCIDIN AS A POSSIBLE MARKER IN DETERMINATION OF MALIGNANCY DEGREE AND SENSITIVITY OF BREAST CANCER CELLS TO CYTOSTATIC DRUGS
}

\author{
T.M. Yalovenko, I.M. Todor, N.Y. Lukianova*, V.F. Chekhun \\ R.E. Kavetsky Institute of Experimental Pathology, Oncologyand Radiobiology, NASof Ukraine, Kyiv 03022, Ukraine
}

Aim: To investigate the role of hepcidin (Hepc) in the formation of cells malignant phenotype in vitro and its expression in the dynamics of growth of Walker-256 carcinosarcoma with different sensitivity to doxorubicin (Dox). Materials and Methods: The cell lines used in the analysis included T47D, MCF-7, MDA-MB-231, MDA-MB-468, MCF/CP, and MCF/Dox. Hepc expression was studied by immunocytochemical method. "Free" iron content was determined by EPR spectroscopy. Determination of Hepc expression in homogenates of tumor tissue and in blood serum of rats with Dox-sensitive and -resistant Walker-256 carcinosarcoma was performed. Results: It was found that Hepc levels in breast cancer (BC) cells with high degree of malignancy (MDA-MB-231, MDAMB-468) and drug-resistant phenotype (MCF/CP, MCF/Dox) were by $1.5-2$ times higher $(p<0.05)$ in comparison with sensitive and less malignant BC cells. The development of drug-resistant phenotype in Walker-256 carcinosarcoma cells was accompanied by increasing of Hepc and "free" iron content (by 2.4 and 1.2 times, respectively). Conclusion: The data of in vitro and in vivo research evidenced on involvement of Hepc in formation of BC cells malignant phenotype and their resistance to Dox.

Key Words: breast cancer, hepcidin, "free" iron, doxorubicin, Walker-256 carcinosarcoma, proliferative activity, invasive potential, adhesion molecules.

Breast cancer $(\mathrm{BC})$ is one of the most common malignancies among the women both in Ukraine and worldwide. Despite the new diagnostic aids and more effective treatment methods, the results of BC treatment remain unsatisfactory. This is due to genetic and morphological heterogeneity of tumors, which is based on the degree of malignancy of tumor cells. According to some studies, the degree of malignancy is a key factor that reflects biological features of tumors. It is proved that increase of the degree of malignancy correlates with the development of metastases, sensitivity to chemo- and hormonal therapy and affects treatment selection, risk of relapses and the quality of life among patients with $B C[1,2]$. Hence, currently there is a need in search for additional informative criteria that will allow us to determine the degree of malignancy and predict the course of BC.

One of the vital problems in experimental and clinical oncology is to overcome tumor resistance to cytostatics. The fact is that no objective criteria, able to determine susceptibility of malignancies to anticancer drugs and consequences of the treatment both for the tumor and the organism, have been found yet [3].

Today it is finally proved that the phenomenon of drug resistance is associated with different intracellular mechanisms of genetic and epigenetic nature [4-8]. At the same time, the list of mechanisms that indicate resistance of malignant cells to different damaging factors is increasing. So, the studies on experimental models in vivo and in vitro contribute greatly to understand the mechanisms, which determine therapy resistance of tumor cells [9-12].

Submitted: May 16, 2016.

*Correspondence: E-mail: lu_na_u@rambler.ru

Abbreviations used: $\mathrm{BC}$ - breast cancer; CP - cisplatin; Dox doxorubicin; Hepc - hepcidin.
In recent years, researchers have focused their attention on the fundamental data regarding the importance of iron in the basic processes of cell activity. Iron is essential and indispensable substance that provides a diversity of biological functions and reactions in the organism and plays a leading role in the pathogenesis of several diseases, including cancer [13-15]. There are data showing that differences in metabolism processes can be observed in drug-sensitive and -resistant tumors [16]. Iron plays an important role in cell growth and division through the proteins involved in the regulation of iron metabolism, oxygen consumption, DNA synthesis and cell death [17]. Today, researchers actively test a hypothesis, according to which ironregulatory proteins are essential at different stages of carcinogenesis, and therefore they can be considered as potential markers of malignant progression and probable targets for anticancer therapy. According to the published results, hepcidin (Hepc) plays one of the main roles among such proteins [18].

According to the data of numerous studies, changes in Hepc expression are observed in a variety of malignant tumors [19-21]. It is shown that the increase of Hepc expression in tumor cells correlates with malignant tumors aggressiveness and low patient survival rates [22, 23]. By contrast, the decrease of Hepc expression inhibits $\mathrm{BC}$ progression by reducing of intracellular iron quantity. Circulating Hepc, which is secreted by liver, has a strong influence on ferroportin-mediated export of iron from the tumor cells. Hepc deficiency increases the outflow of iron from tumor cells and results in repression of tumor growth [24].

Despite the availability of some data on the features of Hepc expression, little is known about Hepc participation in the development of drug-resistant phenotype in malignant tumors. Therefore, there is a need to study Hepc participation in the mechanisms of doxorubicin 
(Dox) resistance in order to develop a strategy for overcoming this phenomenon.

\section{MATERIALS AND METHODS}

Cell lines and drug treatment. The studies were performed in vitro on 6 human BC cell lines: T47D metastatic breast ductal carcinoma; MDA-MB-231 and MDA-MB-468 - metastatic breast adenocarcinoma; MCF-7 - invasive breast ductal carcinoma, MCF-7/ CP, MCF-7/Dox - its variants, resistant to cisplatin (CP) or Dox, respectively.

T47D cellswere cultured in RPMI- 1640 medium (Sigma, USA) supplemented by bovine insulin $(0.2 \mathrm{U} / \mathrm{ml})$ and $10 \%$ fetal bovine serum (FBS). MCF-7 cells were grown in DMEM (Sigma) supplemented by recombinant human insulin $(0.01 \mathrm{mg} / \mathrm{ml})$ and $10 \%$ FBS. MDAMB-231 and MDA-MB-468 cells were cultured in Leibovitz's L-15 medium (Sigma) supplemented by $10 \%$ FBS. All cultures were grown on glass cover slips in humidified atmosphere with $5 \% \mathrm{CO}_{2}$ at $37^{\circ} \mathrm{C}$. The cell lines were obtained from the Bank of Cell Lines from Human and Animal Tissue of the R.E. Kavetsky Institute of Experimental Pathology, Oncology and Radiobiology (IEPOR) of the National Academy of Sciences (NAS) of Ukraine.

The degree of cell malignancy was evaluated by the following criteria: receptor status, proliferation activity, adhesive and invasive properties [25]. MDA-MB-231, MDA-MB-468, MCF-7/CP and MCF-7/Dox cells were considered highly malignant because they did not contain steroid hormone receptors, showed high invasive potential and low adhesive properties. T47D and MCF-7 cells were considered as ones with low degree of malignancy due to high expression of estrogen and progesterone receptors, as well as high adhesive properties due to increased E-cadherin expression and low expression of both $\mathrm{N}$-cadherin and CD44.

The resistant variants MCF-7/Dox and MCF-7/ $\mathrm{CP}$ were originated by growing parental MCF-7 cells with rising concentrations of $\mathrm{CP}$ (from 0.01 to $6 \mu \mathrm{g} / \mathrm{ml}$ ) or Dox (from 0.1 to $32 \mu \mathrm{g} / \mathrm{ml}$ ), respectively. CP and Dox were added twice a week after reseeding. Every 2 months, cell survival rate was analyzed by MTT assay. $I_{50}$ values for MCF-7 and MCF-7/CP cells were 0.25 and $1 \mu \mathrm{g} / \mathrm{ml}$ of $\mathrm{CP}$, respectively, and for MCF- 7 and MCF-7/Dox cells -0.5 and $8 \mu \mathrm{g} / \mathrm{ml}$ of Dox, respectively. Therefore, MCF-7/CP were 4 times as much resistant to the cytotoxic effect of $\mathrm{CP}$ and MCF-7/DOX cells were 16 times as much resistant to the cytotoxic effect of Dox as parental MCF-7 cells.

Immunocytochemical assay. The cells were fixed on cover slips (in triplicate for each sample) in ice-cold methanol:acetone $(1: 1)$ at $-20{ }^{\circ} \mathrm{C}$ for $120 \mathrm{~min}$ and incubated with $1 \%$ bovine serum albumin solution for $20 \mathrm{~min}$. For immunocytochemical assay, primary anti-Hepc monoclonal antibody (1:150) (Abcam, USA). UltraVision LP Detection System (Lab Vision, Thermo Scientific, USA) and DAB Quanto (Thermo Scientific) were used according to the instructions of the manufacturers. When immunocytochemical reaction was completed, the cells were stained with haematoxylin by Mayer and placed in Faramount Aqueous Mounting Medium (DakoCytomation, Denmark). Results were analyzed by light microscopy ( $\times 1000$, oil immersion) with the use of classical $\mathrm{H}$-Score method:

$$
S=1 \cdot N_{1+}+2 \cdot N_{2+}+3 \cdot N_{3+}
$$

where $\mathrm{S}-$ "H-Score" index, $\mathrm{N}_{1+}, \mathrm{N}_{2+}$ and $\mathrm{N}_{3+}-$ number of cells with low, medium or high marker expression [26]. The level of studied markers expression was assigned as follows: low - from 0 to $100 \mathrm{H}$-score points, medium - from 100 to $200 \mathrm{H}$-score points, and high - from 200 to $300 \mathrm{H}$-score points.

Low-temperature Fe(III) electron paramagnetic resonance (EPR). After $24 \mathrm{~h}$ of cultivating, the cells were scrapped, washed in PBS, centrifuged at $1000 \mathrm{~g}$ for $10 \mathrm{~min}$ at $4{ }^{\circ} \mathrm{C}$ and then the pellet was resuspended in PBS. The suspension containing $2 \cdot 10^{6}$ cells was transferred into EPR tubes and immediately frozen in liquid nitrogen. The level of "free" iron was determined by a low-temperature EPR method [27]. Briefly, samples were maintained at $-196{ }^{\circ} \mathrm{C}$ during recording of the spectra using a finger Dewar filled with liquid nitrogen. The following parameters were used for the low-temperature EPR: sweep width $1525 \mathrm{G}$; frequency $9.15 \mathrm{GHz}$; microwave power $40 \mathrm{~mW}$; modulation amplitude $10.0 \mathrm{G}$ and modulation frequency $100 \mathrm{kHz}$. The $g$-value was calculated using the standard formula:

$$
g=h v / \beta H,
$$

where $\mathrm{h}$ - Planck's constant; $\mathrm{v}$ - frequency; $\beta-$ Bohr magneton; $\mathrm{H}-$ external magnetic field at resonance.

Animals and experimental model. The study was performed on female rats (120-150 g weight) bred in the vivarium of the R.E. Kavetsky IEPOR of the NAS of Ukraine (Kyiv, Ukraine). Walker-256 carcinosarcoma was chosen as experimental model. The use and care of the experimental animals have been performed in accordance with the standard international rules of biologic ethics and was approved by Institutional Animal Care and Use Committee.

Tumor transplantation was performed by subcutaneous injection of Walker-256 carcinosarcoma cell suspension $\left(2 \cdot 10^{6}\right.$ cells/animal) in the thigh area. Animals were divided into control (No. 1) and experimental (No. 2) groups (nor less than 10 animals per group). When the volume of tumor reached $0.5 \mathrm{~cm}^{3}$, five Dox (Ebewe, Austria) injections ( $1.5 \mathrm{mg} / \mathrm{kg}$ daily) were administered to the animals of the group No. 2. Then the animals were decapitated under narcosis (Sedazin, Biowet-Pulawy, Poland) according to the rules of Ethic Committee; tumors were resected, tumor cell suspension was prepared and transplanted to the animals from the new control and experimental groups. Then Dox therapy was performed by the same schedule. In total, 12 courses of tumor transplantation/ Dox therapy were performed.

All studies have been performed during development of resistance of Walker-256 carcinosarcoma to Dox. Animals, which underwent removal of tumor for 
the study, have not received cytostatic drug directly. The Hepc level and "free" iron complexes of parental Walker-256 carcinosarcoma (inhibition of growth by Dox has was $65.0 \pm 5.8 \%$ ) and resistant variants of this tumor (inhibition of growth has - $30.0 \pm 2.7 \%$ and $2.1 \pm 0.5 \%$, respectively) have been studied. According with the aim of study, changes in the level of Hepc in blood of animals was determined in different periods of Walker-256 carcinosarcoma growth $-2,5$, 8 and 14 days after tumor transplantation. According to kinetics of tumor growth, the $2^{\text {nd }}-5^{\text {th }}$ days are considered as latent growth period, the $6^{\text {th }}-10^{\text {th }}$ days are referred to as the phase of exponential growth, and the $14^{\text {th }}$ day - as the beginning of the terminal phase of tumor growth [28].

The content of Hepc in blood serum and tumor tissue of experimental animals was measured. In blood and tumors of these animals, "free" iron complexes were determined [29]. Mentioned above indices in tumor were studied on $5^{\text {th }}, 8^{\text {th }}$ and $14^{\text {th }}$ days after tumor transplantation, exactly.

Evaluation of Hepc content in blood serum and tumor homogenates of rats with Walker-256 carcinosarcoma by immunoenzyme method. Content of Hepc in blood serum and tumor homogenate was determined by ELISA using appropriate kits of reagents (ELISA Kit for Hepcidin, USA) with use of automatic biochemical and immunoenzyme analyzer Chem Well 2990 (GBG, USA). Samples of blood serum for ELISA were obtained in accordance with the recommendations indicated in instructions to the kits. All samples of blood serum had no signs of hemolysis. Homogenate of tumor tissue was obtained in PBS solution in the ratio of 1:3.

Statistical analysis. STATISTICA 6.0 computer program (StatSoft Inc., USA) was used for statistical processing of the obtained results. Differences between the average values were compared with use of Student's $t$-test; correlation analysis was performed using Pearson correlation coefficient. Differences were considered as significant with the probability not less than $95 \%(p<0.05)$.

\section{RESULTS AND DISCUSSION}

At the first stage of our study, we analyzed the features of Hepc expression in cells with different degree of malignancy and sensitivity to cytostatics (Table). The level of Hepc expression in tested cell lines varied. The lowest rate of Hepc expression (less than $136 \mathrm{H}$-score points) was observed in the cell lines with low-grade malignancy (MCF-7 and T47D). These lines were characterized by low invasive activity, which according to their molecular phenotype, was determined by increased adhesive properties of these cells due to high E-cadherin expression, low CD44 expression, and the absence of CD24 expression [25]. Indices of $\mathrm{N}$-cadherin expression in the cells with low-grade malignancy were moderate.
Table. Features of Hepc expression in BC cells with various degrees of malignancy and sensitivity to cytostatics

Cell line and degree of malignancy Expression of $\mathrm{Hepc}, \mathrm{H}$-score $(\mathrm{M} \pm \mathrm{m})$ points T47D, low $(\mathrm{n}=3) \quad 97.0 \pm 1.3$

MCF-7, low $(\mathrm{n}=3) \quad 136.0 \pm 3.1$

MDA-MB-231, high $(n=3) \quad 246.0 \pm 1.9$

MDA-MB-468, high $(\mathrm{n}=3) \quad 311.0 \pm 2.6$

MCF-7/CP, high $(\mathrm{n}=3) \quad 220.0 \pm 2.8$

MCF-7/Dox, high $(\mathrm{n}=3)$

$207.0 \pm 1.7$

High Hepc expression ( $\geqslant 200 \mathrm{H}$-Score points) was observed in the cells with high degree of malignancy (MDA-MB-231, MDA-MB-468). In contrast to T47D and MCF-7 cell lines with low invasive potential, these cells had the highest rate of invasive activity, which was determined by significantly higher expression of CD44 and CD24 adhesion molecules.

In addition, we evaluated the features of Hepc expression in two drug-resistant $\mathrm{BC}$ cell lines such as MCF-7/CP and MCF-7/Dox (see Table). According to the results of these studies, Hepc expression in MCF7/CP and MCF-7/Dox cell lines was much higher than in cells with low-grade malignancy. It should be mentioned that the rates of invasiveness in both resistant cell lines were similar to the rates in cells with high degree of malignancy. Both drug-resistant cell lines demonstrated low rates of E-cadherin expression, high rates of $\mathrm{CD} 44$ and $\mathrm{N}$-cadherin expression, and the absence of CD24 [25]. So, the in vitro studies demonstrated that the increase in Hepc expression level is associated with a high degree of malignancy and the absence of sensitivity of human BC cells to $\mathrm{CP}$ and Dox.

Resistance to anticancer therapy is one of the vital problems in the modern oncology. Primarily, this is due to the fact that today there is no comprehensive data on all the rearrangements, which occur in tumor and tumor-bearing organism during the development of resistance. Despite the availability of some information about the features of Hepc expression in malignant processes, there are no published findings regarding Hepc participation in the formation of a malignant phenotype of cells, which are resistant to cytostatics. Also, there are no data regarding the changes in iron homeostasis in tumor and in the organism during the growth and progression of drug-sensitive and -resistant strains of malignant neoplasms. That is why, at the second stage of the research we studied levels of "free" iron and Hepc in tumor and organism during the formation of resistance to Dox in dynamics of growth of sensitive and resistant Walker-256 carcinosarcoma.

We studied these parameters in tumor cells of sensitive and resistant Walker-256 carcinosarcoma starting from the $5^{\text {th }}$ day after tumor transplantation (end of latent period). As seen from the data presented in Fig. 1, the development of drug-resistant phenotype in Walker-256 carcinosarcoma cells was accompanied by increasing of Hepc and "free" iron content ( 2.4 and 1.2 times, respectively). 

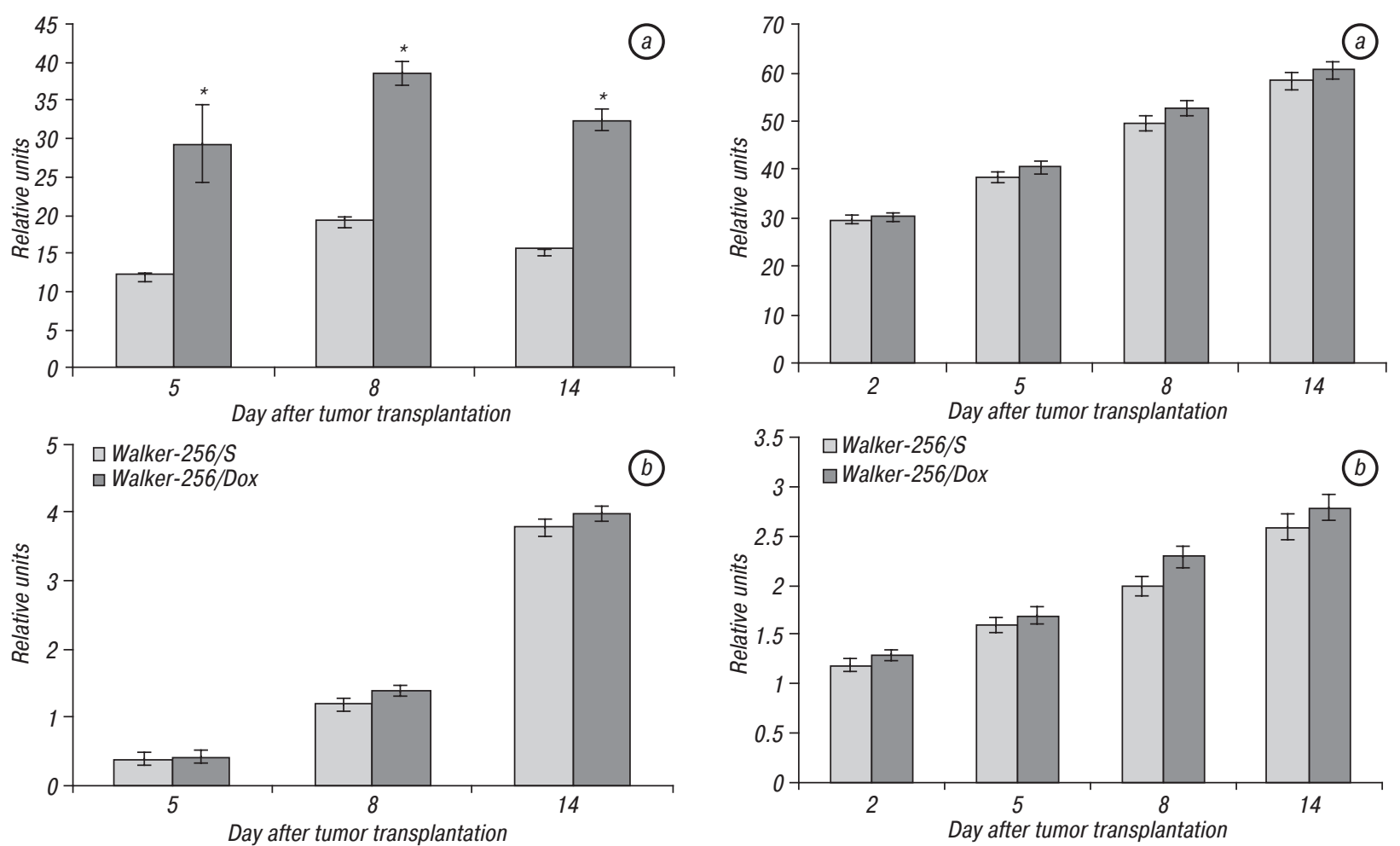

Fig. 1. Changes of Hepc (a) and "free" iron $(b)$ levels in tumor cells during development of resistance to Dox and the growth of sensitive (Walker-256/S) and resistant (Walker-256/Dox) Walker-256 carcinosarcoma $(n=10)$. Note: ${ }^{*} p<0.05$ compared to the sensitive strain of Walker-256 carcinosarcoma

The study of Hepc levels during the growth of experimental tumors showed that the maximum content of the protein in tumor was observed at the exponential phase of growth ( $8^{\text {th }}$ day; Fig. 1$)$ and in blood serum - in terminal phase (14 ${ }^{\text {th }}$ day; Fig. 2). Reduction of Hepc content in tumor tissue at the terminal stage of tumor growth ( $14^{\text {th }}$ day), in our opinion, is explained by the prevalence of necrobiotic changes and necrotic processes. This proved the fact of intensification of metabolic processes associated with a higher Hepc content in the tumor tissue of animals with more aggressive tumor phenotype. At the same time, we found out that the growth and progression of sensitive and resistant Walker-256 carcinosarcoma is accompanied by significant increase in the level of "free" iron in tumor cells (see Fig. 1). Thus, during the exponential and terminal phases of growth, the level of "free" iron in the cells of both strains is, respectively, 3 and 9 times higher than during the latent phase. The established fact corresponds to the known data about iron as a key element for cell proliferation and growth [30]. So, in our study we found out that changes of Hepc and "free" iron levels in tumor cells during growth of sensitive and resistant Walker-256 carcinosarcoma are of identical nature.

The analysis of the studied parameters in the organism showed some changes in the Hepc and "free" iron content in the serum of animals with drug-sensitive and -resistant tumor (Fig. 2). We found that the growth of sensitive and resistant Walker-256 carcinosarcoma is accompanied by same changes in the content of "free" iron levels in serum of experimental animals.

Fig. 2. Content of Hepc (a) and "free" iron $(b)$ complexes in serum of animals during the growth of sensitive (Walker-256/S) and resistant (Walker-256/Dox) Walker-256 carcinosarcoma

There was increase in Hepc level in the serum while its maximum level was observed in terminal phases of tumor growth ( 14 days after tumor transplantation). It should be noted that Hepc level in the blood of animals with resistant strains increased more rapidly compared to the animals with sensitive Walker-256 carcinosarcoma. These differences can probably be explained by the increased requirement of drug-resistant tumor cells in Hepc.

So, the development of resistance to Dox in vivo was accompanied by changes in "free" iron and Hepc levels in tumor and organism of tumor-bearing animals. These changes of studied parameters depended on the growth phase of experimental tumors. Our findings suggested that there were some correlations between Hepc expression and such indices of BC malignancy as receptor status, adhesive and invasive properties of cells. In particular, Hepc expression depended on the invasion level of $B C$ cells with low and high degree of malignancy. According to results of numerous studies about Hepc participation in iron metabolism and the role of iron in the division and growth of cells [13, 14, 31-33] our findings indicated a direct role of Hepc in formation of malignant phenotype and resistance of cells to drug treatment.

In addition, our study demonstrated the same character of changes in the levels of "free" iron and Hepc in tumor and host during the growth of sensitive and resistant Walker-256 carcinosarcoma. We showed that the development of resistance to Dox in Walker-256 carcinosarcoma in vivo was accompanied by increase of Hepc and "free" iron levels. These facts coincide with the available data [33-35] and 
confirm the importance of Hepc in iron metabolism in tumor cells for development of drug resistance and progression. So, both in vitro and in vivo we proved that Hepc is involved in development of a malignant phenotype of BC cells and their drug resistance to cytostatic drugs. The obtained data extend the existing knowledge about the role of Hepc in $\mathrm{BC}$ progression highlighting the importance of further studies on Hepc relevance in cancer progression and prognosis.

\section{REFERENCES}

1. James L, Khatcheressian, Hurley P, et al. Breast cancer follow-up and management after primary treatment: American Society of Clinical Oncology clinical practice guideline update. J Clin Oncol 2013; 31: 961-5.

2. Harris LN, Ismaila N, McShane LM, et al. Use of biomarkers to guide decisions on adjuvant systemic therapy for women with early-stage invasive breast cancer: American Society of Clinical Oncology clinical practice guideline. J Clin Oncol 2016; 34: 1134-50.

3. Liang XJ, Chen C, Zhao Y, Wang PC. Circumventing tumor resistance to chemotherapy by nanotechnology. Methods Mol Biol 2010; 596: 467-88.

4. Chekhun VF, Shishova YV. Current view on the mechanisms of drug resistance of tumor. Oncology 2000; 2: 11-5 (in Russian).

5. Bible KC, Boerner SA, Kirkland K, et al. Characterization of an ovarian carcinoma cell line resistant to cisplatin and flavopiridol. Clin Cancer Res 2000; 6: 661-70.

6. Ding Z, Yang X, Pater A, Tang SC. Resistance to apoptosis is correlated with the reduced caspase- 3 activation and enhanced expression of antiapoptotic proteins in human cervical multidrug-resistant cells. Biochem Biophys Res Commun 2000; 270: 415-20.

7. Huang L, Snyder AR, Morgan WF. Radiation-induced genomic instability and its implications for radiation carcinogenesis. Oncogene 2003; 22: 5848-54.

8. Kumar P, Miller AI, Polverini PJ. P38 MAPK mediates gamma-irradiation-induced endothelial cell apoptosis, and vascular endothelial growth factor protects endothelial cells through the phosphoinositide 3-kinase-Akt-Bcl-2 pathway. J Biol Chem 2004; 279: 43352-60.

9. Housman G, Byler S, Heerboth S, et al. Drug resistance in cancer: an overview. Cancers (Basel) 2014; 6: 1769-92.

10. Grigorovich SA, Brenner B, Svirnovski AI. Original contributions factors supporting survival in multidrug resistant lymphoblast cell line IM-9/ER. Exp Oncol 2001; 23: 242-7.

11. Svirnovski A, Pasiukov V. Attempts to influence the drug resistance of tumor cells in experimental system. Exp Oncol 2005; 27: 43-6.

12. Uchiyama-Kokubu N, Watanabe T. Establishment and characterization of adriamycin-resistant human colorectal adenocarcinoma HCT-15 cell lines with multidrug resistance. Anticancer Drugs 2001; 12: 769-79.

13. Chekhun VF, Shpilevaya SI. Role of endogenous iron in the formation of tumor sensitivity to anticancer therapy. Vopr Onkol 2010; 56: 251-60 (in Russian).

14. Elliot RL, Head JF. Cancer: tumor iron metabolism, mitochondrial dysfunction and tumor immunosuppression; "a tight partnership - was Warburg correct?". J Cancer Ther 2012; 3: 278-311.

15. Lavrova VS, Chernova NI, Karpova GV, et al. Disregulating processes in the blood system during cancer. Bull Sib Med 2006; 5 (2): 75-89 (in Russian).
16. Chen Y, Yu G, Yu D, Zhu M. PKC $\alpha$-induced drug resistance in pancreatic cancer cells is associated with transforming growth factor- $\beta 1$. J Exp Clin Cancer Res 2010; 29: 104.

17. Zhang C. Essential functions of iron-requiring proteins in DNA replication, repair and cell cycle control. Protein Cell 2014; 5: 750-60.

18. Rossi E. Hepcidin - the iron regulatory hormone. Clin Biochem Rev 2005; 26: 47-9.

19. Kijima H, Sawada T, Tomosugi N, Kubota K. Expression of hepcidin mRNA is uniformly suppressed in hepatocellular carcinoma. BMC Cancer 2008; 8: 167.

20. Esfandi F, Mohammadzadeh Ghobadloo S, Basati G. Interleukin-6 level in patients with colorectal cancer. Cancer Lett 2006; 244: 76-8.

21. Kamai T, Tomosugi $\mathbf{N}$, Abe $\mathbf{H}$, et al. Increased serum hepcidin-25 level and increased tumor expression of hepcidin mRNA are associated with metastasis of renal cell carcinoma. BMC Cancer 2009; 9: 270.

22. Chen Q, Wang L, Ma Y, et al. Increased hepcidin expression in non-small cell lung cancer tissue and serum is associated with clinical stage. Thorac Cancer 2014; 5: 14-24.

23. Torti SV, Torti FM. Ironing out cancer. Cancer Res 2011; 71: 1511-4.

24. Guo W, Zhang S, Chen Y, et al. An important role of the hepcidin-ferroportin signaling in affecting tumor growth and metastasis. Acta Biochim Biophys Sin (Shanghai) 2015; 47: 703-15.

25. Chekhun VF, Zalutskii IV, Naleskina LA, et al. Modifying effects of lactoferrin in vitro on molecular phenotype of human breast cancer cells. Exp Oncol 2015; 37: 181-6.

26. McClelland RA, Wilson D, Leake R. A multicentre study into the reliability of steroid receptor immunocytochemical assay quantification. Eur J Cancer 1991; 27: 711-5.

27. Pate KT, Rangel NA, Fraser B, et al. Measuring "free" iron levels in Caenorhabditis elegans using low-temperature $\mathrm{Fe}(\mathrm{III})$ electron paramagnetic resonance spectroscopy. Anal Biochem 2006; 358: 199-207.

28. Emanuel NM. The kinetics of experimental tumor processes. Moscow: Nauka, 1977. 419 p. (in Russian).

29. Vashchenko G, MacGillivray RT. Multi-copper oxidases and human iron metabolism. Nutrients 2013; 5: 2289-313.

30. Heath JL, Weiss JM, Lavau CP, Wechsler DS. Iron deprivation in cancer - potential therapeutic implications. Nutrients 2013; 5: 2836-59.

31. Park CH, Valore EV, Waring AJ, Ganz T. Hepcidin, a urinary antimicrobial peptide synthesized in the liver. J Biol Chem 2001; 276: 7806-10.

32. Krause A, Neitz S, Mägert HJ, et al. LEAP-1, a novel highly disulfide-bonded human peptide, exhibits antimicrobial activity. FEBS Lett 2000; 480: 147-50.

33. Nguyen NB, Callaghan KD, Ghio AJ, et al. Hepcidin expression and iron transport in alveolar macrophages. Am J Physiol Lung Cell Mol Physiol 2006; 291: L417-25.

34. Pinnix ZK, Miller LD, Wang W, et al. Ferroportin and iron regulation in breast cancer progression and prognosis. Sci Transl Med 2010; 2: 43ra56.

35. Winand FJ, Boegemann M, Gallitz I, et al. GDF15 and hepcidin as prognostic factors in patients with prostate cancer. J Mol Biomark Diagn 2014; 5: 199. 